\title{
The effect of electron-electron correlation on the attoclock experiment
}

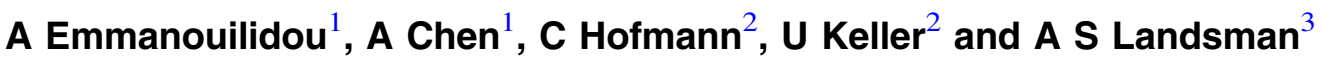 \\ ${ }^{1}$ Department of Physics and Astronomy, University College London, Gower Street, London WC1E \\ 6BT, UK \\ ${ }^{2}$ Department of Physics, ETH Zurich, Zurich, Switzerland \\ ${ }^{3}$ Max Planck Institute for the Physics of Complex Systems, Noethnitzer Strasse 38, Dresden, Germany \\ E-mail: a.emmanouilidou@ucl.ac.uk
}

Received 26 May 2015, revised 3 September 2015

Accepted for publication 9 September 2015

Published 23 October 2015

\begin{abstract}
We investigate multi-electron effects in strong-field ionization of helium using a semi-classical model that, unlike other commonly used theoretical approaches, takes into account electronelectron correlation. Our approach has an additional advantage of allowing to selectively switch off different contributions from the parent ion (such as the remaining electron or the nuclear charge) and thereby investigate in detail how the final electron angle in the attoclock experiment is influenced by these contributions. We find that the bound electron exerts a significant effect on the final electron momenta distribution that can, however, be accounted for by an appropriately selected mean field. Our results show excellent agreement with other widely used theoretical models done within a single active electron approximation.
\end{abstract}

Keywords: semiclassical model, strong field ionization, attoclock experiment

The idea of the attoclock with a clearly defined 'time-zero' for strong field ionization was first introduced in [1], and relies on elliptical polarization of an ultra-short laser pulse to map the final offset angle of the electron momenta distribution to time. Since the attoclock technique extracts the center of the electron momenta distribution (figure 1), the accuracy is limited in principle only by the electron statistics at the detector, hence enabling the reconstruction of electron dynamics in the attosecond $\left(10^{-18} \mathrm{~s}\right)$ domain. However, as many reconstruction and experimental calibration procedures in strong field ionization experiments [2-4], the measured delay time relies on a semi-classical (two-step) model within the single active electron approximation [5-8].

Recent experiments $[6,7]$ found an additional angular offset, relative to an adiabatic two-step model, which takes account of the Coulomb field of the parent ion within a single active electron approximation (for details on the model see $[5,9,10])$. This additional offset was explained as due to tunneling delays, following the attoclock concept, as presented in $[1,7,11]$. A subsequent work [8] showed that using an imaginary time method developed by Perelomov, Popov, and Terent'ev (PPT) [12], which takes account of non-adiabatic effects but neglects the Coulomb tail inside the potential barrier, gives essentially the same interpretation of the experimental result in [7] as the above mentioned adiabatic model. On the numerical front, there remains a discrepancy between time-dependent Schrödinger equation (TDSE) simulations performed within the single active electron approximation $[13,14]$ and the experimental results in $[6,7]$. It has therefore been suggested that this discrepancy potentially can be explained by the presence of electron-electron correlations in helium.

Another recent experiment has shown that electronelectron correlations can play an important role in excited helium atoms when ionized with an ultra-violet photon [15]. Most recently, however, excellent agreement was obtained for attosecond photoemission measurements from ground state helium with three different theoretical models, which go from ab-initio theory taking into account both electrons to singleactive electron approximation with both the TDSE and a fully analytical model [16]. A question remains as to whether such correlation effects are significant in the present experimental range of the attoclock, which involves strong field tunnel ionization (using a laser pulse with central wavelength in the infrared range) of helium from a ground state. Resolving this question with TDSE simulations of a two electron atom in the 


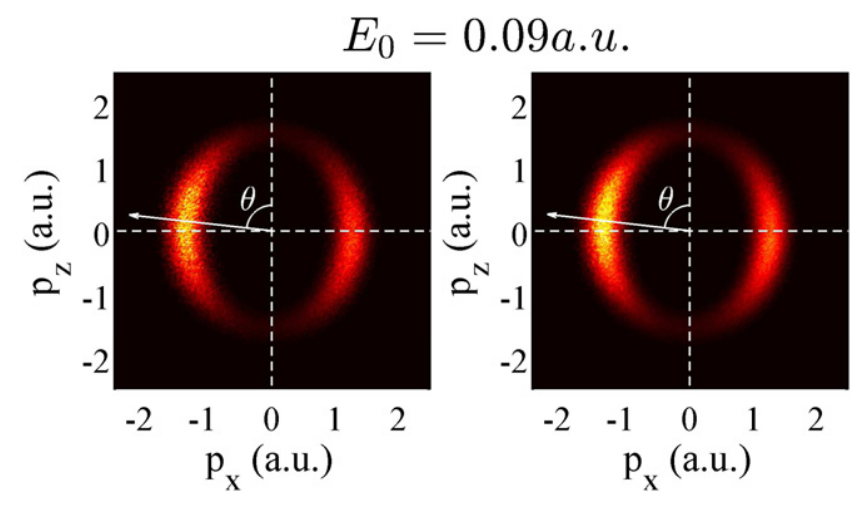

Figure 1. The offset angle for the three-body Coulomb system (left) and for the two-body system with an effective charge $Z=1$ (right).

relevant experimental regime is a formidable task. We therefore use a semi-classical two electron model, introduced in [17], that allows for a consistent description of the process, taking into account the initial bound state and the propagation in the continuum following tunneling.

Since the current two-electron model, like other semiclassical models, assumes instantaneous tunneling, it can be used as a calibration for the attoclock experiment, which aims to extract delays associated with the tunneling process $[1,7]$. In particular, this model can be used to calculate the contributions to the measured angle incurred after the electron appears at the tunnel exit. The difference between this calculated contribution and the experimentally measured angle (see figure 3) can then be used to extract delays associated with the tunneling process (such as was done in [7] using a semi-classical single active electron model introduced in [5]).

To study the effect of electron-electron interaction on the 2D momentum distribution of the singly ionizing electron in $\mathrm{He}$ in an elliptically polarized laser field (i.e. the experimental observable in $[6,7,18])$, we employ a three-dimensional (3D) semi-classical model for a three-body Coulomb system (two electrons plus the nucleus). All interactions are fully accounted for including electron-electron correlation. The quasiclassical model we use entails one electron (electron 1) instantaneously tunneling through the field-lowered Coulomb potential with a quantum tunneling rate given by the Ammosov-Delone-Krainov formula [9, 19, 20]. The longitudinal momentum is zero while the transverse one is given by a Gaussian distribution [19]. We compute the exact exit point for the tunneling electron using parabolic coordinates [10]. The remaining electron (electron 2) is modeled by a microcanonical distribution [21]. For the evolution of the classical trajectories we use the full three-body Hamiltonian in the laser field, that is

$H=\frac{p_{1}^{2}}{2}+\frac{p_{2}^{2}}{2}-\frac{Z}{r_{1}}-\frac{Z}{r_{2}}+\frac{1}{\left|\mathbf{r}_{1}-\mathbf{r}_{2}\right|}+\left(\mathbf{r}_{1}+\mathbf{r}_{2}\right) \cdot \mathbf{E}(t)$,

with $\mathbf{E}(t)$ the laser field. An important difference between our $3 \mathrm{D}$ model and other 3D semi-classical models $[10,22]$ is that our model treats exactly the Coulomb singularity by introducing regularized coordinates [23] for the time propagation. Transforming to regularized coordinates removes the Coulomb singularity due to the electron-nucleus interaction. This results in a faster and more stable numerical integration. The model we currently employ was first described in [17] and was proven to accurately describe several strong field phenomena in atoms driven by infrared laser pulses [17, 24].

Our goal is to compute the offset angle of the $2 \mathrm{D}$ electron momentum distribution in the plane of polarization as a function of the intensity of the laser field and compare with the experimental results in $[6,7]$. To do so, we model the electric field using a Gaussian pulse, similar to the one employed in the experiments that are of interest for the current work [5-7, 18, 25]:

$$
\mathbf{E}(t)=E_{0} f(t)(\cos \omega t \hat{z}+\epsilon \sin \omega t \hat{x})
$$

with

$$
f(t)=\exp \left(-2 \ln 2\left(\frac{t}{t_{\mathrm{FWHM}}}\right)^{2}\right)
$$

and $t_{\mathrm{FWHM}}=6 \mathrm{fs}, \omega=0.05695$ a.u., with $E_{0}$ the amplitude of the laser field. We use the ellipticity parameter $\epsilon=0.87$. These laser pulse parameters were selected to model the experimental set-up described in [6,7]. The use of the dipole approximation in equation (2) allows one to neglect the spatial dependence of the laser field as well as the magnetic fields, which would otherwise considerably complicate the dynamics [26], hence allowing for an easier and more intuitive interpretation of classical trajectories [27]. The initial time of propagation is sampled randomly from $\phi_{0}=\omega t_{0}=$ [ $-34,34]$ a.u., with $\phi_{0}$ the initial phase of the laser field at the time electron 1 tunnels.

We compute the 2D, $x-z$, momenta distributions of the ionizing (tunneling) electron, in single ionization events, for different laser field strengths. Specifically, we consider the field strengths $0.03,0.05,0.07,0.09,0.11$ and 0.13 a.u. In figure 1 , we plot the 2D momentum distribution for a field strength of $E_{0}=0.09$ a.u. We extract the offset angle from these momenta distributions as follows. For each singly ionizing event we register the final momentum of the tunneling electron, which escapes to the continuum. From the final momentum we compute the angle $\theta=\arctan \left(p_{x} / p_{z}\right)$ and the magnitude of the momentum $p=\sqrt{p_{x}^{2}+p_{z}^{2}}$. The distribution $S(\theta, p)$ denotes the probability for the singly ionizing electron to escape with angle $\theta$ and momentum $p$, respectively. Integrating over the magnitude of the momentum, we then obtain the distribution $f(\theta)$ that denotes the probability for an electron to singly ionize with angle $\theta$ :

$$
f(\theta)=\int S(\theta, p) p \mathrm{~d} p .
$$

We use a double gaussian function to fit $f(\theta)$, i.e. the same function as the one used to fit the experimental results in $[6,7]$. It was shown in prior work [28] that, for high ellipticities, the radial integration method described above 
Table 1. The offset angle for the three-body Coulomb system (left) and the two body Coulomb system with an effective charge $Z_{\text {eff }}=1$ (right).

\begin{tabular}{lcc}
\hline$E_{0}$ (a.u.) & Three-body $\left({ }^{\circ}\right)$ & Two-body, $Z_{\text {eft }}=1\left(^{\circ}\right)$ \\
\hline 0.03 & 8.4 & 8.4 \\
0.05 & 6.1 & 6.2 \\
0.07 & 5.8 & 6.0 \\
0.09 & 6.1 & 6.3 \\
0.11 & 6.6 & 6.9 \\
0.13 & 6.8 & 7.2 \\
\hline
\end{tabular}

integrates exclusively over the transverse momenta spreads of the electron distribution at the detector.

Using the above described procedure, we extract the maximum, $\theta_{\max }$, of the $f(\theta)$ distribution, which corresponds to the most probable value of the angle $\theta$. We then identify $90^{\circ}$ $-\theta_{\max }$ as the streaking offset angle (see figure 3 ). $90^{\circ}$ is how much the momentum at the end of the pulse is offset compared to the laser field at the time of tunneling, assuming no tunneling delays and neglecting the Coulomb interaction with the parent ion. This can be understood by considering that, neglecting interaction with the parent ion, the final electron momentum is given by the vector potential at the instant, $t_{0}$, that the electron appears at the tunnel exit: $\mathbf{p}_{\text {final }}=\mathbf{A}\left(t_{0}\right)$. Hence, the electrons which appear at the tunnel exit at $t=0$, will acquire final momentum $\mathbf{p}_{\text {final }}=\mathbf{A}(0)$, which corresponds to a rotation of $90^{\circ}$ with respect to the major axis of polarization (the value of $\mathbf{A}(0)$ can be easily calculated from the electric field given in equation (2)).

First, we investigate whether, following tunneling, the electron-electron interaction in the three-body Hamiltonian can be accounted for by using a two-body Coulomb system (one electron plus the nucleus) with an effective nuclear charge equal to $Z_{\text {eff }}=1$. In figure 1 , we plot the $2 \mathrm{D}$ momenta distributions for $E_{0}=0.09$ a.u. for the three-body and the two-body Coulomb systems. The two distributions are very similar. Indeed, in table 1 we list the offset angles $90^{\circ}-\theta_{\max }$ for different strengths of the laser field for the three-body and the two-body systems. This similarity of the two sets of values implies that the electron-electron interaction can be accounted for by a one electron plus a nucleus system with an effective charge. This suggests that following tunneling the core potential does not significantly affect the momenta distributions. The reduced influence of the core potential is consistent with the tunneling electron emerging from the barrier on the order of 10 a.u. away from the core; this is the case even for the largest field strengths considered in this work.

We now investigate how each contribution in the threebody Hamiltonian affects the delay time. First, switching off the electron-electron interaction, we compute the delay time due to the effect of the nucleus with $Z=2$ on the tunneling electron, see table 2 and figure 3 (dashed blue curve). Next, we compute the effect of electron-electron correlation on the dynamics of the ionized electron. Specifically, we compute the offset angle when we switch off the electron-nucleus
Table 2. The offset angle for the three-body Coulomb problem as in table 1 (a), for the three-body problem but with the interaction of the tunneling electron with the nucleus switched off (b) and for the twobody problem with $Z=2$.

\begin{tabular}{lccc}
\hline$E_{0}$ (a.u.) & $\begin{array}{c}\text { Three-body } \\
\text { (a) }\left({ }^{\circ}\right)\end{array}$ & $\begin{array}{c}\text { Three-body } \\
\text { (b) }\left(^{\circ}\right)\end{array}$ & $\begin{array}{c}\text { Two-body, } \\
Z=2\left(^{\circ}\right)\end{array}$ \\
\hline 0.03 & 8.4 & -7.1 & 17.1 \\
0.05 & 6.1 & -5.5 & 12.9 \\
0.07 & 5.8 & -5.2 & 11.8 \\
0.09 & 6.1 & -5.2 & 12.3 \\
0.11 & 6.6 & -4.9 & 12.7 \\
0.13 & 6.8 & -4.6 & 13.9 \\
\hline
\end{tabular}

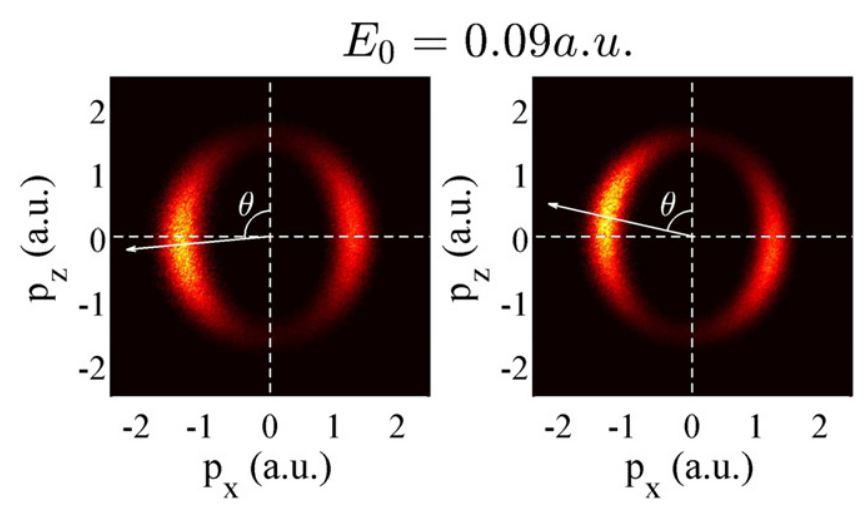

Figure 2. The offset angle for the three-body Coulomb system when the electron-nucleus interaction is switched off for the tunneling electron (left) and for the two-body system with $Z=2$ (right).

interaction following tunneling. In this case, the offset angle we compute will be due to the effect of the Coulomb repulsion that the tunneling electron experiences from electron 2 that remains bound. In figure 2, we show for a fixed field strength that as a result of the electron-electron interaction the offset angle is in the $p_{z}<0$ and $p_{x}<0$ quadrant unlike the offset angles for the cases of the full three-body system and the two-body system that are in the $p_{z}>0$ and $p_{x}<0$ quadrant. Specifically, as shown over a wide range of electric field strengths in table 2 and figure 3, while for the full threebody and the two-body system the offset angles $90^{\circ}-\theta_{\max }$ are positive, when we switch off the electron-nucleus interaction on the tunneling electron the offset angle is negative (see figure 3, dashed red line). This implies that the Coulomb repulsion between the bound electron and the tunneling electron causes the latter to escape to the continuum faster than it would in free-space. The above implies that the electron-electron interaction (in the mean field sense) does have a significant effect on the dynamics of the tunneling electron while escaping to the continuum. Table 2 also shows the large positive offset angle due to the effect of the nucleus on the tunneling electron. This positive offset angle is reduced by the effect of the bound electron on the tunneling electron (negative offset angle), resulting in a smaller positive offset angle for the three-body Hamiltonian where all interactions are accounted for. Our results outside the barrier show that the electron-electron correlation causes the tunneling electron to 


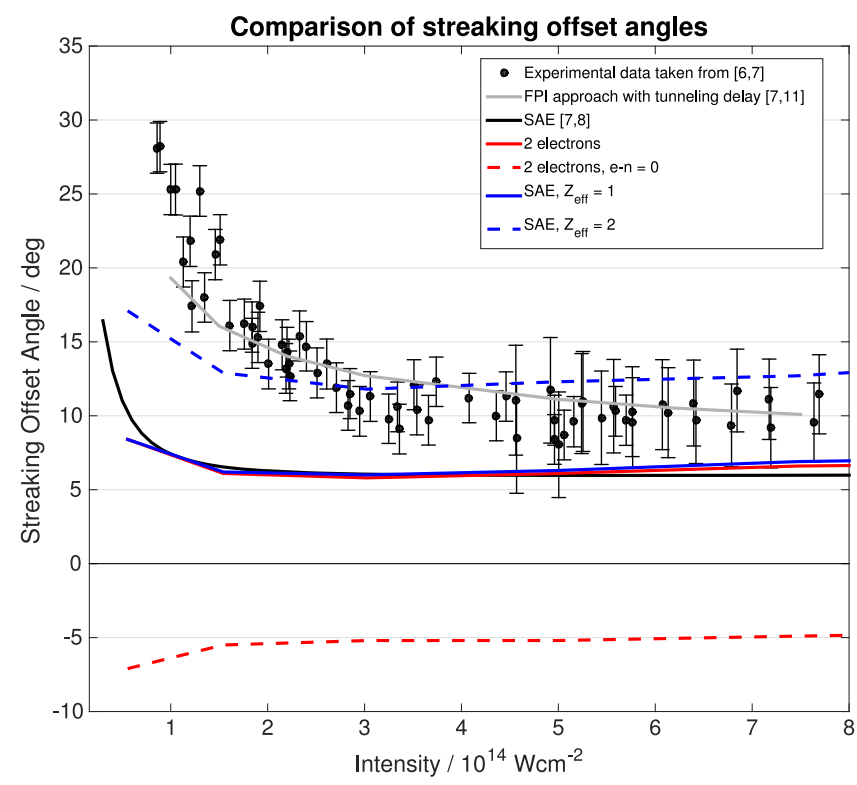

Figure 3. Comparison of semi-classical models to experimental results. All semi-classical models are within a single active electron approximation, except for the solid and dashed red lines. The colored dashed lines are the result of selectively switching off either the nuclear charge (dashed red line) or the charge of the remaining electron (dashed blue line).

escape faster to the continuum resulting in a decreased offset angle. While our model does not account for the dynamics inside the barrier, based on our results and given that the tunneling electron is a valence electron, we conjecture that it is likely that the electron-electron correlation inside the barrier will also result in the tunneling electron escaping faster. If this is the case, electron-electron correlation can not account for the experimental offset angle being significantly higher than the theoretical one.

Figure 3 shows that the offset angle obtained using a semi-classical model (solid red line) is significantly smaller than the experimental one, particularly for small intensities. Can electron-electron correlation account for this discrepancy? We believe this not to be the case for the following reasons: so far we have shown that, within the framework of our model, the offset angle is the same whether we fully account for two electron effects or in a mean-field sense. This can be seen in figure 3, where the two electron (three-body system) results (solid red line) almost coincide with the single active electron approximation (solid blue line), when the effective charge of the remaining parent ion is such that $Z_{\text {eff }}=1$. (In regard to the single active electron model, the effective charge before the ionization takes place has to be greater than one, since the nuclear charge in a ground state of helium is only partially shielded by the other electron.)

However, we note that our theoretical results are obtained with a model which does not consider the dynamics inside the barrier. It is only after electron 1 exits the barrier that we propagate in time accounting for all the interactions among the two electrons and the nucleus as well as the interactions with the laser field. Figure 3 compares our results (solid red and blue lines) with experimental data and with another adiabatic model described in [22] and referred to as TIPIS in [5] (solid black line in figure 3). As can be seen, there is good agreement between the adiabatic semi-classical models over a wide intensity range in which the attoclock experiments in $[6,7]$ were performed.

Interestingly, for the experimental parameters plotted in figure 3, there is also a very good agreement between our adiabatic two-electron model and non-adiabatic models developed within a single active electron approximation using the imaginary time method, in particular the well-known PPT [12], and the recent related approach called the ARM model [29, 30], developed by Smirnova and co-workers. The agreement between TIPIS (solid black line in figure 3) and PPT (not shown) was already demonstrated in [8]. By extension, the ARM model is a perturbative correction to PPT and predicts a similar final offset angle to PPT-see the discussion section IV B of [30], which explains that the 'initial coordinate in the ARM model is essentially the same as in the nonadiabatic short-range theory' (referring to PPT). It follows that, for the experimental results shown in figure 3, our two-electron model agrees well with both adiabatic and non-adiabatic semi-classical models developed within a single active electron approximation.

It is instructive to make a more detailed comparison of the results of the simulations with the recent experimental data, taken from [7] and plotted in figure 3. In particular, the gap between the semi-classical models and the experimental data is on the order of $10^{\circ}$ at lower intensities, corresponding to times as high as 100 attosecond [7] (the angle is mapped to time, such that $\tau=\delta \theta / \omega$, where $\delta \theta$ corresponds to the gap between the measured and the calculated angles). Since, as noted above, the semi-classical models agree in their predictions of the final offset angle, the interpretation of this gap between experiment and semi-classical simulations is a matter of debate that hinges on the significance of multi-electron effects during the tunneling process itself: if the multi-electron effects are negligible or subtract from the final angle (as a classical repulsive interaction would do), then the gap between the measurement and the semi-classical models indicates a non-negligible tunneling delay time, as was concluded in [7].

Investigating rigorously electron-electron correlations during the tunneling process itself (while the electron is inside the barrier) would be the next step in ruling out multi-electron effects in the attoclock experiments. However, such studies are beyond the scope of existing semi-classical models. Fully quantum, two-electron, TDSE simulations using a t-SURFF method [31] are currently in the planning stages [32]. Such simulations could definitively resolve the importance of electron-electron correlations in helium, during strong field ionization from a ground state, by comparing TDSE simulations within a single active electron approximation [13], with a full two-electron TDSE solution.

In conclusion, we note that taking account of electronelectron correlations after tunneling within a semi-classical model cannot account for the additional angular offset measured in the recent attoclock experiments $[6,7]$. In particular, 
a careful use of the single active electron approximation results in essentially the same angle for the center of the electron momenta distribution as the use of a full two electron model. One of the advantages of our two-electron model is that it allows for a consistent description of the entire process, both the initial bound state and the subsequent propagation of the ionized electron in the continuum. On the other hand, the single active electron approximation requires that we use different effective charge for the initial bound atomic state (where it must be such that it reproduces the ionization potential of helium) and for the propagation in the continuum, where the total effective charge of the parent ion should be set to one. Using the two-electron model also allowed us to investigate the relative contributions of the nucleus and the remaining bound electron to the final offset angle.

\section{Acknowledgments}

A E acknowledges support from EPSRC under Grant No. J0171831 and use of the Legion computational resources at UCL. U K acknowledges ERC advanced grant ERC-2012ADG_20120216 within the seventh framework programme of the European Union and A S L and U K acknowledge the support by the Swiss National Science Foundation (SNSF) project grant Nr. 200021_153432. A S L acknowledges the Max Planck Center of Attosecond Science (MPC-AS).

\section{References}

[1] Eckle P et al 2008 Science 3221525

[2] Shafir D et al 2012 Nature 485343
[3] Meckel M et al 2008 Science 3205882

[4] Arissian L et al 2010 Phys. Lev. Lett. 105133002

[5] Pfeiffer A N et al 2012 Nat. Phys. 876

[6] Boge R et al 2013 Phys. Rev. Lett. 111103003

[7] Landsman A S et al 2014 Optica 1343

[8] Landsman A S and Keller U 2014 J. Phys. B 47204024

[9] Landau L D and Lifschitz E M 1965 Quantum Mechanics Nonrelativistic Theory 2nd edn (Oxford: Pergamon)

[10] Hu B, Liu J and Chen S G 1997 Phys. Lett. A 236533

[11] Landsman A S and Keller U 2015 Phys. Rep. 547 1-24

[12] Perelomov A M, Popov V S and Terent'ev M V 1966 JETP 501393

[13] Hofmann C et al 2014 Phys. Rev. A 90043406

[14] Ivanov I A and Kheifets A S 2014 Phys. Rev. A 89 021402(R)

[15] Stodolna A S et al 2014 Phys. Rev. Lett. 113103002

[16] Heuser $S$ et al 2015 arXiv:1503.08966 submitted

[17] Emmanouilidou A 2008 Phys. Rev. A 78023411

[18] Landsman A S et al 2013 Phys. Rev. Lett. 111263001

[19] Delone N B and Krainov V P 1991 J. Opt. Soc. Am. B 81207

[20] Ammosov M V, Delone N B and Krainov V P 1986 Sov. Phys. -JETP 641991

[21] Abrines R and Percival I C 1966 Proc. Phys. Soc. London 88861

[22] Fu L B, Liu J, Chen J and Chen S G 2001 Phys. Rev. A 63 043416

Fu L B, Liu J and Chen S G 2002 Phys. Rev. A 65 021406(R)

[23] Kustaanheimo P and Stiefel E 1965 J. Reine Angew. Math. 218204

[24] Emmanouilidou A, Parker J S, Moore L R and Taylor K T 2011 New J. Phys. 13043001

[25] Landsman A S et al 2013 New J. Phys. 15013001

[26] Ludwig A et al 2014 Phys. Rev. Lett. 113243001

[27] Corkum P B 1993 Phys. Rev. Lett. 711994

[28] Hofmann C et al 2013 J. Phys. B 46125601

[29] Torlina L et al 2015 Nat. Phys. 11503

[30] Kaushal J and Smirnova O 2013 Phys. Rev. A 88013421

[31] Tao L and Scrinzi A 2012 New J. Phys. 14013021

[32] Scrinzi A 2015 private communication 